\title{
¿Qué hacer para tener buenos precios de la luz?
}

\section{Pedro Linares}

Universidad Pontificia Comillas - ICAI

E-mail: pedrol@comillas.edu

Recibido: 5 de octubre de 2021 Aceptado: 14 de octubre de 2021

RESUMEN: La fuerte subida de los precios de la electricidad experimentada en los últimos meses en Europa, y en particular en España, ha generado, además de malestar social, una acalorada discusión sobre las medidas políticas necesarias para atajarla. En este artículo evalúo las principales propuestas, y propongo una serie de principios generales para tratar de que el precio de la electricidad sea justo y eficiente, analizando cada uno de sus componentes.

PALABRAS CLAVE: electricidad; precios; regulación; pobreza energética.

\section{What to do to get good electricity prices?}

ABSTRACT: The significant increase in the price of electricity that Europe and particularly Spain has been suffering in the last months has produced, besides social unrest, a heated discussion about the policy measures required to mitigate it. In this short paper, I assess the main proposals, and I put forward a set of general principles that should be followed for the electricity price to be fair and efficient, looking at each of its components.

KEYWORDS: electricity; prices; regulation; energy poverty.

\section{Introducción}

La electricidad está disparada en toda Europa. La razón principal es la escalada de precios del gas natural y del $\mathrm{CO}_{2}$ en sus mercados correspondientes, que se traslada a una subida del precio final que llega a los consumidores acogidos a la tarifa regulada, el conocido PVPC. Se han propuesto varias soluciones, pero la mayoría de ellas parten de una consideración fragmentada, y políticamente viciada, de cómo funciona el mercado eléctrico. Aquí las repaso una a una, aprovechando el viaje para considerar los aspectos fundamen- 
tales de dicho mercado, y cómo deberíamos diseñar el sistema de fijación de precios para que sea, al mismo tiempo, justo y fiel a los costes que debería reflejar.

Pero, antes de comenzar, debo decir que he dudado mucho si escribir esto en estos momentos, porque creo que una reflexión como la que planteo en el artículo sólo puede hacerse desde la tranquilidad, y no desde la urgencia y ruido que hay estos días. Pero ahora que el Gobierno ya ha hecho sus propuestas, creo que ya tiene sentido dar mi opinión. En todo caso, creo que muchos de los temas que discuto a continuación sólo pueden abordarse desde un debate tranquilo y riguroso, y con tiempos amplios para su implantación y el consenso suficiente. Ojalá que llegue ese momento, que no parece llegar nunca en la historia reciente del sector eléctrico español.

¿Qué podemos hacer para tener unos buenos precios de la luz? Lo primero de todo, no querer tocarlos. Es decir, diseñar un sistema en el cual la tarifa se calcule, no se fije, como siempre pide el profesor Ignacio Pérez-Arriaga. Eso supone, primero, diseñar una regulación que dé las señales adecuadas a los agentes (más sobre eso después), y segundo, que el Gobierno renuncie a intervenir en los mercados cada vez que hay quejas, por ejem- plo, dando todas las competencias a la CNMC.

A este respecto, hay gente que defiende que el dejar en manos de un regulador, que no se presenta a elecciones, el control de los precios de la luz, es una pérdida democrática. Yo no estoy de acuerdo, por dos razones: primero, porque a los reguladores los elige el Parlamento, que representa la voluntad popular no necesariamente peor que el Gobierno; segundo, porque no veo qué ventaja para esto tiene el que los políticos, llevados de los ciclos electorales, sean los que vayan acumulando medidas de corto plazo, muchas veces erróneas. De hecho, los propios políticos deberían darse cuenta de que si el coste de apuntarse la medalla de bajar la luz (que todos desgraciadamente buscan, o demandan desde la oposición, según dónde estén) es todo el lío que se monta alrededor, como estos días, quizá no compense.

Pero entonces, ¿qué hacemos si sube como estos días el precio del gas y el precio del $\mathrm{CO}_{2}$ ? Si tenemos un buen diseño (que explico más abajo), lo único que tendríamos que hacer sería proteger a los consumidores vulnerables (después comento más). Porque el hecho de que la tarifa refleje los costes reales es, en general, bueno para el consumidor medio. 
En tanto no tenemos ese buen diseño, como nos pasa ahora, y si la subida de precios es tan elevada que llega a afectar también a las rentas medias, la única propuesta urgente que creo razonable es enviar un cheque para las familias y empresas que consideremos que necesitan ayuda (y que no son todas, evidentemente), por el exceso de gasto en el que están teniendo que incurrir. Este instrumento es temporal y acotado, no distorsiona los mercados, y no crea problemas distributivos como hacen las bajadas de impuestos.

Pero esto como digo debe ser sólo una medida puntual y limitada. A medio plazo lo que hay que hacer es analizar, y diseñar, el sistema de forma integral. Para explicar esto voy a tomar prestado también de Ignacio Pérez Arriaga el método didáctico "de la cebolla": empezaré por las ideas más sencillas, y luego las iré matizando y desarrollando más.

\section{2. ¿Debemos fijar el precio por ley?}

La propuesta más radical es, por desgracia, también la más intuitiva: si el precio de la luz es excesivo, el Estado debería tener la capacidad de fijarlo en un nivel más limitado. Pero, en realidad, hay tres razones centrales para desear que los precios de electricidad reflejen los costes de la producción energética.

Primero, porque damos la señal correcta de eficiencia: si el gas es caro y además tiene que pagar $\mathrm{CO}_{2}$, incentivamos a los consumidores a ahorrar (no sólo energía, sino también emisiones), y a los productores a instalar otras tecnologías más limpias y más baratas, como las renovables, o como el almacenamiento, sin tener que recurrir a sistemas de apoyo que no siempre funcionan bien. Y esto, a medio y largo plazo, es bueno para todos.

Segundo, porque animamos a los consumidores a protegerse frente a la volatilidad de los precios, firmando contratos a largo plazo, que es algo que todos echamos de menos en los mercados para que funcionen mejor. De hecho, lo normal es que las industrias intensivas en energía, que son las que más podrían sufrir ante un repunte de precios, tengan su suministro cubierto en gran medida frente a este tipo de riesgos.

Tercero, porque a pesar del incendio mediático que se ha montado estos días, hay que recordar que estamos hablando de cantidades perfectamente gestionables por un hogar o una industria media, 
sobre todo si los ponderamos a lo largo del año (por ejemplo, en 2021 hemos tenido unas cuantas horas con precios irrisorios o nulos). A este respecto, estamos hablando de que una subida del $20 \%$ de la tarifa eléctrica anual del hogar medio se traduce en 10-12 euros de variación mensual, y eso si no adoptamos medidas de ahorro. ¿Cuánto suben las gasolinas o las tarifas de móviles, que tienen más incidencia en el presupuesto de los hogares, y cuyas estructuras productivas son similares a las de la electricidad, sin que digamos nada? Por supuesto, habrá hogares y empresas que no puedan permitirse esto: a esos habrá que ayudarles, con cheques/transferencias como decía antes, y no con descuentos o bajadas de impuestos.

En cambio, ¿qué pasa si fijamos o ponemos un tope a la tarifa? Pues que lo que dejemos de pagar al llegar a este tope lo tendrán que pagar otros (o nosotros mismos en un par de años). El déficit de tarifa que llevamos años pagando nos debería desanimar a utilizar este tipo de soluciones (la que ha activado el Gobierno para la tarifa regulada de gas, por cierto). Además, el tope o el control de precios de la electricidad o el gas, igual que la bajada de impuestos, tiene una derivada distributiva no de- seable: beneficia más a los hogares que más energía consumen, los más ricos.

Mi conclusión inicial, pues, es que deberíamos mantener nuestro sistema de mercado mayorista, igual que se mantiene en $\mathrm{Eu}-$ ropa, y dejar las tarifas reguladas exclusivamente para los hogares vulnerables, para los que de verdad necesitan protección. Y si hace falta extender la protección a las rentas medias, usemos los cheques de forma focalizada para los que realmente lo necesitan.

\section{La composición del precio de la luz}

Voy ahora con la segunda pasada, en la que describo los elementos que creo que habría que tener en cuenta para asegurarnos de que los precios de la electricidad recogen sus costes, y nada más. $\mathrm{Al}$ final también hablaré de la protección a los vulnerables.

El precio de la luz tiene cuatro componentes: generación, peajes de transporte y distribución, cargos, e impuestos. Su peso en el recibo de la luz del mes actual de un hogar medio se recoge en la figura siguiente: 


\section{¿Qué hacer para tener buenos precios de la luz?}

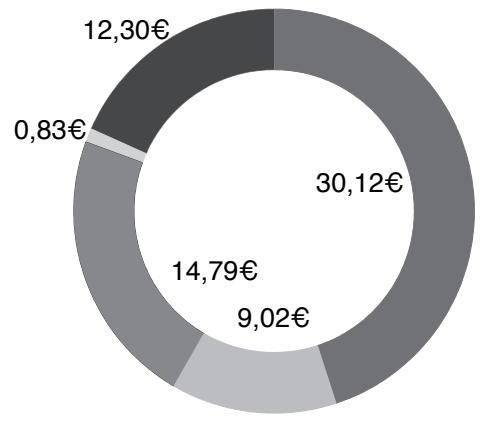

RECORE:5,90€

\section{DÉFICIT: 4,66€}

Impuestos:

Alquiler Contador:

Energía:

Cargos:

Peajes de Transporte y Distribución
$9,02 €$

$0,83 €$

$30,12 €$

$12,30 €$

$14,79 €$
Vamos a ver qué podríamos hacer para lograr que cada uno recoja lo que cuesta, y nada más.

\section{El coste de generación}

Primero, el coste de generación, determinado por el tan denostado mercado marginalista (tan marginalista como cualquiera de los productos que compramos habitualmente) en función de los costes de producción de electricidad con las distintas tecnologías.

Lo primero que podríamos tratar de hacer sería reducir el coste de producción de la electricidad. El problema es que esto, habitualmente, está en gran medida fuera de nuestro control. Por ejemplo, el coste del gas viene determinado por el mercado internacional. Pero lo que sí podríamos es tratar de modificar el mix de generación para utilizar tecnologías menos volátiles. Si vamos introduciendo cada vez más renovables, tal como está previsto, el coste no sabemos si bajará o no (dependerá de la evolución de los costes de las renovables y del respaldo necesario), pero sí sabemos que será más estable, al depender fundamentalmente del coste de la inversión y no del combustible. $Y$ además tampoco tendremos que pagar $\mathrm{CO}_{2}$. Algo similar pasaría con la nuclear, de no abandonarse como está previsto.

La segunda posibilidad es revisar el mercado, para asegurarnos de que recoge correctamente los costes. El mercado marginalista, recordemos, tiene como ventaja que envía las señales correctas a los inversores: cuando sube el precio, el incentivo a invertir aumen- 
ta, y más aún para las tecnologías inframarginales (como las renovables). Esta señal puede venir tanto del precio del combustible marginal (el gas) o del precio del $\mathrm{CO}_{2}$ : al subir el precio del $\mathrm{CO}_{2}$ las tecnologías limpias son más rentables. Eso es justo lo que se ha demandado mucho tiempo, cuando el precio del mercado europeo era "demasiado" bajo.

El problema del mercado marginalista es que puede sobrerretribuir a tecnologías "agotadas", es decir, las que no necesitan esa señal porque no se va a invertir más en ellas por distintas razones, y que no han realizado inversiones que deban recuperarse en un entorno liberalizado. En estos casos, el precio de mercado es más bien una transferencia de renta, no una señal de eficiencia. Si esto se une a que, en España, la mayor parte de estas tecnologías fueron en muchos casos construidas con otro régimen retributivo (el marco legal y estable), ya tenemos el lío. Frente a este lío hay dos opciones:

Una, la que ha usado el Gobierno, aplicar un "windfall tax", un impuesto sobre los beneficios caídos del cielo. Para mí esta opción tiene muchos problemas, y el principal es que seguramente no sobreviva a las reclamaciones legales (incluso con medidas cautelares) que se derivarán de la pérdida que supone para todos los que tienen contratada de antemano la energía (muchos generadores, muchas comercializadoras), porque además diferenciar entre estos contratos es muy complejo. $\mathrm{Y}$ si sobrevive al corto plazo, no me sorprendería que la termináramos pagando unos años después. Javier Revuelta explica muy bien los problemas de corto plazo con los contratos y las tarifas fijas que podrían hacer que algunas empresas pequeñas quebraran. Más problemas: no tiene sentido que esta minoración aplique a las tecnologías construidas en régimen liberalizado (y por tanto a riesgo, tanto de precios bajos como altos); y calcular la minoración a realizar es muy complicado (¿qué precio del gas o del $\mathrm{CO}_{2}$ usamos como referencia?). Como digo, una vía muy difícil que salga adelante.

A mí me parece más sencillo y robusto, en el caso en que se determinara que estas tecnologías están efectivamente agotadas, y cuando no se hubieran construido en régimen liberalizado, determinar una retribución razonable para ellas, y firmar contratos por diferencias (algo que ya proponía Ignacio Pérez Arriaga en el Libro Blanco), de forma que recuperen sus costes (incluidas las inversiones no amortizadas), pero nada más. A este respecto, es importante recor- 
dar que entre los costes que tendría que recuperar la nuclear están su desmantelamiento completo y la gestión de los residuos, algo que ahora no está garantizado, y menos con el cierre anticipado. Respecto a la hidráulica, también es importante mencionar que un contrato por diferencias mantendría la señal de operación óptima: que la hidráulica entre en el mercado cuando los precios son mayores. Eso sí, esta vía no se puede utilizar con carácter de urgencia, por todos los problemas mencionados anteriormente: habría que diseñarla cuidadosamente, negociarla con todos los agentes, y establecer un período de transición.

En el caso de las hidráulicas, hay una opción más, que además es mejor si se puede o cuando se pueda utilizar: si se sacan a subasta las concesiones, se eliminaría el problema de los posibles beneficios extraordinarios, porque se internalizaría el potencial beneficio en la puja.

El otro problema, más reciente, es que el mercado marginalista "energy-only" no parece capaz de retribuir a las renovables. Esto también tiene solución: dejarlo como un mercado de ajustes, y establecer un mercado de capacidad (o mejor, opciones de fiabilidad) o de renovables que asegure la retribución a este tipo de tecnologías. O estimular la contratación a plazo. En esta línea, la propuesta del Gobierno de obligar a que las empresas tengan que vender parte de su producción a plazo puede ayudar a crear este mercado (además de a reducir la posibilidad de poder de mercado).

En todo caso, y a pesar de esta solución, hay gente empeñada en eliminar el mercado marginalista (a pesar del pequeño problema de la normativa europea) y sustituirlo de nuevo por el marco legal y estable, el sistema regulado que tuvimos hasta 1998 por el cual el Estado definía las inversiones y retribuía a los operadores del sistema. Ni siquiera muchas empresas rechazan esto, porque evidentemente les libera del riesgo inversor, que se traslada completamente al consumidor. ¿Es más barata esta solución que un mercado ajustado como he mencionado antes? Pues no necesariamente, porque un marco totalmente regulado, como ya hemos experimentado en épocas pasadas, tiene un importante incentivo a sobreinvertir (de nuevo, porque los errores los paga el consumidor, no el tecnócrata que puede equivocarse decidiendo la cantidad que hay que construir de cada tecnología). Es decir, la diferencia es quién corre con el riesgo, pero no necesariamente el coste. 
Más allá de esto, otra posible razón para que el mercado no recoja bien los costes es que exista poder de mercado, y se ejerza por parte de los agentes. Esto es otro tema bastante controvertido, pero se supone que para vigilar esto, y evitarlo, están los reguladores, tanto el europeo (ACER) como el español, la CNMC, que de hecho ya ha llevado en ocasiones a empresas a los tribunales cuando ha detectado comportamientos ilícitos.

\section{El coste de las redes}

El coste de las redes se minimiza si los inversores (Red Eléctrica y las distribuidoras) tienen el incentivo a optimizar su inversión y operación. Actualmente, esto no sucede, porque la retribución se establece en función de la inversión, y no de una gestión eficiente. Así que todas las empresas tienen un claro incentivo a sobreinvertir, porque ya lo pagaremos los consumidores. Los topes exógenos, como el que existe actualmente, no son buena solución, porque impiden invertir cuando realmente hace falta. Sería mucho mejor utilizar un sistema como el británico, que retribuye la eficiencia en la inversión y el uso de las redes.

En este coste de las redes también habría que incluir el coste de las interconexiones, que como ya he comentado otras veces, tienen ventajas e inconvenientes, y que por tanto deberían ser optimizadas (no vale cuanto más mejor).

\section{Los cargos}

Tercero, los cargos (es decir, el coste de las políticas públicas o la financiación del déficit de tarifa anterior). Aquí hay dos cuestiones: una, cuánto tenemos que pagar, y otra, de qué forma hacerlo.

Lo primero es únicamente relevante a futuro. Lo pasado, pasado está, y hay que recuperarlo sí o sí. Lo futuro, evidentemente debería decidirse con buenos criterios y buen análisis, incluyendo por supuesto buena comunicación a la sociedad de las ventajas e inconvenientes de las políticas propuestas. Esta es la única forma de lograr que su coste sea el adecuado, $\mathrm{y}$ de que sean aceptadas.

Y luego: ¿deben recuperarse los costes de estas políticas públicas en la tarifa eléctrica, o repartiéndolos entre los distintos vectores energéticos (como propone el FNSSE), o en los Presupuestos Generales del Estado (PGE)? Esta pregunta debe responderse fundamentalmente mirando quién se beneficia de la política en cuestión, si sólo los consumidores eléc- 
tricos, los energéticos, o todos los contribuyentes. Es cierto que, ahora mismo, en la tarifa hay elementos, como las extrapeninsulares o la pobreza energética, que seguramente debieran estar completamente en los PGE. Otros, como el coste de las renovables, puede tener sentido repartirlos entre todos los consumidores energéticos, aunque también se podría defender su paso a PGE.

Hay otra cuestión adicional aquí relacionada con el reparto: cuando diseñamos los cargos de forma que hay subsidios cruzados a la eficiencia energética o al autoconsumo, unos consumidores se benefician y otros pagan de más. Esto evidentemente también hay que tenerlo en cuenta a la hora de diseñar el sistema que permita pagar lo justo.

\section{Los impuestos}

Los impuestos, tanto los aplicados sobre la generación como los que van sobre la tarifa. Aunque esto debe estarlo evaluando la comisión de expertos sobre fiscalidad, me atrevo a lanzar algunas ideas:

La primera de hecho aplica también a los cargos (que no son tan distintos conceptualmente en algunos casos de los impuestos): hay argumentos sólidos para defender que los impuestos (o algunos cargos) no deberían aplicarse cuando se utiliza la electricidad como bien intermedio. Esto ya sucede, por diseño, con el IVA, pero no con otros. Si se siguiera esta regla, se abarataría la electricidad para las empresas, pero quizá se encareciera para los hogares (salvo que los cargos se pasen a PGE).

Hay gente que incluso defiende que habría que eliminar toda la fiscalidad de la electricidad, también para los hogares. Esto ya me parece más complejo, para empezar porque no sé qué razones hay para pensar que esta actividad económica no deba contribuir a la recaudación de los impuestos necesarios para que funcione nuestra sociedad: ¿por qué, por ejemplo, quitar impuestos a la electricidad y no a las tarifas de telefonía móvil? Por tanto, parece razonable suponer que el IVA debería mantenerse (su potencial reducción también es un tema complejo y no evidente), y también parece razonable establecer impuestos que internalicen las distintas externalidades de la producción y uso de la electricidad. Más allá de eso, sería necesario un análisis global de las necesidades de recaudación y de las alternativas disponibles.

En cualquier caso, es importante recordar una cuestión: si hace fal- 
ta recaudar para financiar el gasto público, el reducir los impuestos en un producto o servicio supone simplemente que otro producto o servicio tendrá que pagar más, con las consecuencias correspondientes en la (mala) asignación de recursos en la economía. Esto de hecho es lo que pasará con algunas de las medidas propuestas por el Gobierno: las reducciones de impuestos suponen menos recaudación. Si esa recaudación era extraordinaria (una especie de beneficios caídos del cielo también para Hacienda), no pasa nada por eliminarla en tanto esos beneficios extraordinarios existan; pero si hace falta para financiar los gastos públicos, tendrá que venir de otro sitio.

Una vez hemos diseñado un sistema que asegure que los costes son los justos, faltaría asegurar que las comercializadoras, en un mercado libre (porque como ya hemos dicho la tarifa regulada debería aplicarse sólo a los vulnerables), trasladan adecuadamente el precio a los consumidores. De nuevo, la CNMC debe vigilar que no haya abuso de mercado por parte de las comercializadoras, y debe dar transparencia (como ya hace en su comparador de ofertas) a los precios. En estas condiciones, cada consumidor elegirá si quiere tener una factura más estable (pagando la prima de riesgo correspondiente) o si quiere estar más expuesto a los precios de mercado, que, mientras sigamos teniendo gas en el sistema, serán volátiles.

\section{La protección de los vulnerables}

Como ya hemos defendido en otras ocasiones, y sigue haciendo la Cátedra de Energía y Pobreza del ICAI-Universidad Pontificia Comillas ${ }^{1}$, la protección de los vulnerables debería incluir tanto el apoyo a la renta (mediante un cheque energético, con una cantidad a tanto alzado que les permita cubrir su suministro imprescindible) como la prioridad en las actuaciones de eficiencia energética. El sistema actual del bono eléctrico, basado en un descuento del precio, no protege totalmente a estos hogares (ya que tienen que seguir pagando el $75 \%$ del aumento de precios). Otra alternativa sería una tarifa regulada, que evidentemente no podría ser como el PVPC (que no protege a los vulnerables) sino más estable, y seguramente, subsidiada.

1 https://www.comillas.edu/catedrade-energia-y-pobreza 


\section{9. ¿Sirven para algo las medidas propuestas desde el Gobierno o la oposición?}

Algunas de las medidas recientemente introducidas por el Gobierno en el RDL 17/2021 ya las he comentado antes, ahora voy con el resto.

Lo de la bajada del IVA no es solución más allá del corto plazo: para las empresas, como el IVA es neutro, esto sólo ayuda en la gestión de la tesorería; para los hogares, el bajar el IVA aquí supone que habrá que subirlo en otros sitios si queremos mantener la recaudación (salvo que, como he dicho antes, sea recaudación extraordinaria). Y además hay que recordar que esta medida es regresiva: se benefician más los más ricos, que son los que consumen más electricidad.

Lo mismo puede decirse de la detracción de los ingresos de las subastas de $\mathrm{CO}_{2}$, es desvestir a un santo para vestir a otro: en este caso, la innovación y la promoción de tecnologías limpias, o la protección a los consumidores vulnerables, que es para lo que se supone que estaba esto.

Las subastas obligatorias se han usado en otros países más como una medida para reducir el poder de mercado que como instrumen- to para reducir costes. Podrían funcionar como alternativa a los contratos por diferencias regulados administrativamente, pero siempre que la demanda no sea tan alta que el precio acabe siendo similar al del mercado. Además, es muy importante recordar que la hidráulica debe seguir teniendo una señal eficiente de operación, como es el coste de oportunidad de la térmica a la que sustituye, porque si no estaremos gestionando mal el agua escasa. En todo caso, si la subasta obligatoria se traduce en mayor contratación a plazo, esto sí puede ayudar a que la demanda de este tipo de contratación aumente.

Por último, la propuesta de la empresa pública: si es una comercializadora como las que se han creado en algunos ayuntamientos, podrá tratar de reducir su margen, pero seguirá teniendo que pagar el resto de los costes. Y si ofrece precios por debajo del coste, esto supone un subsidio que habrá que financiar. Así que creo que por ahí no hay mucho que rascar. Si es una empresa pública de generación que produce electricidad con las hidráulicas cuya concesión vaya caducando, tampoco arreglamos mucho: como decía antes, la hidráulica debe ofrecerse al coste de oportunidad para que su gestión sea la apropiada, si no, 
podemos estar produciendo con la hidráulica en las horas en que el precio está bajo, que es cuando no hace falta, y no produciendo cuando los precios son altos, que es cuando de verdad se la necesita. Si el objetivo de la empresa es vender electricidad producida con hidráulica a precio de coste, ¿qué incentivo tendrá a ofertarla al coste de oportunidad, que es la forma que mejor gestiona el agua? Y si realmente se hiciera así, entonces estas hidráulicas recibirían la misma renta que ahora, renta que habría que modificar igual que si la operación es priva- da. De nuevo, tampoco veo una ventaja particular al hecho de que sea pública, incluso tendría como inconveniente una potencial reducción de incentivos a gestionar bien el agua.

Termino como empecé: creo que todo esto hay que debatirlo tranquilamente, con transparencia, rigor, y tiempo. Las prisas no son buenas, porque acaban llevando a despropósitos y populismos. Ojalá se pueda reconducir la conversación hacia ese espacio de sosiego que tanta falta hace, igual que las reformas ${ }^{2}$.

2 https://economicsforenergy.wordpress.com/ 